\title{
Doxorubicin Induced Histomorphometric Changes in Testes of Albino Rats
}

\author{
Surendra Kumar Sah ${ }^{1}$, Saroj Khatiwada ${ }^{2 *}$, Deepak Chaudhary ${ }^{1}$, Chandra Bhushan Jha ${ }^{3}$, \\ Soumya Bhattacharya ${ }^{3}$ \\ ${ }^{1}$ Department of Anatomy, Nobel Medical College, Biratnagar, Nepal \\ ${ }^{2}$ Department of Biochemistry, Modern Technical College, Lalitpur, Nepal \\ ${ }^{3}$ Department of Human Anatomy, B P Koirala Institute of Health Sciences, Dharan, Nepal
}

\begin{abstract}
Anticancer drugs like doxorubicin have been found to affect male gonads thereby leading to infertility. This study was conducted to evaluate the effects of doxorubicin over short, mid and long term on testes of male albino rats. Sixty male albino rats aged 6-8 weeks were taken for study. The rats were randomly divided into 3 groups of experimental (each group containing 10 rats) and 3 groups of control (each group containing 10 rats). The experimental groups were given a single dose of doxorubicin i.e. $10 \mathrm{mg} / \mathrm{kg}$ body weight intra-peritoneally and sacrificed after 3 different duration for each group (second week, eighth week and sixteenth week). All rats under 3 control groups were given a single intra-peritoneal dose of $2.5 \mathrm{ml} / \mathrm{kg}$ body weight normal saline and sacrificed with their respective experimental groups. Significant difference in diameters $(p=0.029)$ and cross-sectional area $(p=0.028)$ of seminiferous tubules was observed between short term experimental and short term control rats. For both between midterm experimental and midterm control group, and between long term experimental and long term control group, a significant difference in right testis weight ( $p<0.001$ for both), left testis weight ( $p<0.001$ for both), volume of testis $(p<0.001$ and $p=0.038)$, diameter $(p<0.001$ for both) and area $(p<0.001$ for both) of seminiferous tubules was observed. As compared to short term experimental group, midterm experimental group and long term experimental group had significantly lower right testis weight ( $\mathrm{p}<0.001$ for both), left testis weight $(\mathrm{p}<0.001$ for both), diameter of seminiferous tubule $(\mathrm{p}<0.001$ for both) and cross-sectional area of seminiferous tubule ( $\mathrm{p}<0.001$ both). Cross-sections of the seminiferous tubules of all the control groups had normal architecture. However, there was progressive destruction of seminiferous tubules structure across the experimental groups. Doxorubicin has deleterious effect on seminiferous tubules of albino rat testis.
\end{abstract}

Key words: Doxorubicin, Histomorphometry, Seminiferous tubule, albino rat

*Corresponding author

Email: khatiwadasaroj22@gmail.com

\section{Introduction}

Doxorubicin, also known as hydroxyd-aunorubicin, is a drug used in cancer chemotherapy. It is highly effective in many human tumors and is currently the first line anti-cancer drug in many chemoresponsive tumors such as ovarian cancers, breast cancers and lymphomas [1]. The clinical use of doxorubicin can be viewed as double edged sword. On one hand, doxorubicin plays an undisputed key role in the treatment of many neoplastic diseases; on the other hand, chronic administration of doxorubicin induces organ toxicity particularly testicular injury [2].

Previous investigations indicated that doxorubicin has the ability to induce mutations and chromosomal aberrations in normal and malignant cells in addition to a wide variety of toxic side effects on organs like testes [3]. Doxorubicin is said to alter sperm development, production, structural integrity and motility rates in association with increased cellular apoptosis in spermatogonia and spermocyte [4]. The reduction of oxidative DNA damage by antioxidants has been evaluated as a chemotherapeutic approach for reducing damage caused by chemotherapy agents such as doxorubicin [5].

Damage to the testicular germinal epithelium is a potential side effect of cancer therapy, and is of particular concern in case of men of reproductive age having tumors with high cure rates [6]. Thus it seems important to assess the effects of doxorubicin on various components of male reproductive system and particularly in testis where sperm production takes place. Different studies have been carried out to investigate the effects of doxorubicin on testis, however there are only few studies utilizing stereological tool to elucidate histomophometric 
evidences of doxorubicin mediated derangement of the testis and evaluate midterm and long term effects of doxorubicin [7]. Considering this we conducted the present study among albino rats to find short, mid and long term effects of doxorubicin on histomorphometric characters of testis as compared to controls.

\section{Methods}

The study was conducted at the department of anatomy of B P Koirala Institute of Health Sciences, Dharan, Nepal to find the short, mid and long term effects of doxorubicin on testis of albino rat as compared to controls. Sixty healthy albino rats aged 6-8 weeks and weighing 150-200 gm were obtained from the animal house of anatomy department and used in study. They were housed in well ventilated room at controlled ambient temperature $\left(25 \pm 5{ }^{\circ} \mathrm{C}\right)$ with a 12 hour alternating light-dark cycle and provided standard rodent diet and water throughout the study period. The experimental work was carried out as per research and ethical guidelines of Nepal Health Research Council (NHRC) for the care and use of animals in health research in Nepal and the research protocol was approved by the institute review board of B P Koirala Institute of Health Sciences.

The rats were randomly divided into 3 groups of experimental (each group containing 10 rats) and 3 groups of control (each group containing 10 rats), such that weight do not vary by more than $10 \%$ of average weight of study population. The experimental groups were given a single dose of doxorubicin i.e. $10 \mathrm{mg} / \mathrm{kg}$ body weight intraperitoneally and sacrificed after 3 different duration for each group. Group 1 animals, which were investigated for short term effect, were sacrificed on 14th day (2nd week). Group 2 animals (studied for midterm effects) were sacrificed on 56th day (8th week), and group 3 animals (studied for long term effects) on 112th day (16th week). All rats under 3 control groups (control 1, control 2 and control 3) were given a single intra-peritoneal dose of 2.5 $\mathrm{ml} / \mathrm{kg}$ body weight normal saline and sacrificed with their respective experimental groups (at second, eighth and sixteen weeks respectively). Comparison was done among 3 groups of experimental rats in terms of effect of doxorubicin, and as well as between each matched experimental and control group.

All rats were weighed at the time of euthanasia. Animals were anesthetized with ether soaked in cotton and their testes were fixed by in vivo perfusion technique as mentioned below. Rats were kept on the dissecting tray with their ventral surface facing upward and all four limbs pinned. The abdomen was opened to expose the abdominal aorta and inferior vena cava. A 18 guage needle was inserted on the abdominal aorta and tied with thread to keep it in constant position. The needle was attached to clean flask tube, connected with two bottles containing bouin's fluid and physiological saline separately. Then the chest was opened and the right atrium was nicked by a scissor or knife (scalpel) to permit the drainage of blood. Thereafter, the physiological saline was perfused to flush out the blood. The fixative bouin's fluid was then transfused with the help of three ways stopcock. Approximately $200 \mathrm{ml}$ of each fluid was perfused. Perfusion was stopped when clear fixative drops started oozing out from the snout of the animal. After completion of perfusion, the testes were isolated from the scrotum with the help of scalpel and forceps and weight was measured followed by volume measurement by the application of water displacement method and post fixed for 24 hours by the same fixative.

After 18 hour fixation in bouin's fluid, the tissue was processed and histological slides were prepared from vertical sections from the polar and the equatorial regions of each testis. Micrometer was used for quantitative measurement of parameter; diameter of seminiferous tubules, and interstitial spaces, germ cells, sertoli cells and leydig cells were observed qualitatively in histological slides. Diameter (D) of each seminiferous tubule was measured by two directions and mean of two was taken as a diameter of that seminiferous tubule. The cross-sectional area of the seminiferous tubules were determined by formula; Area $(\mathrm{A})=\Pi \mathrm{D}^{2} / 4$. Two slides per rat were observed for both control and experimental groups.

Statistical analysis was done in SPSS version 19. All data were expressed as mean \pm SD. Independent $t$ test and one way ANOVA was applied to test for statistical significance at $95 \%$ confidence interval. A 
value of $\mathrm{p}<0.05$ was considered statistically significant.

\section{Results}

It was observed that mean body weight consistently decreased among the short term and long term rats. On the other hand, there was increase in body weight among the midterm rats in comparison to its initial body weight, as well as in controls. Various anatomical parameters in experimental groups and controls are shown in table 1.

No significant difference in right testis weight $(\mathrm{p}=0.184)$, left testis weight $(\mathrm{p}=0.179)$, and testicular volume $(p=0.388)$, but significant difference in diameters $(\mathrm{p}=0.029)$ and cross-sectional area $(p=0.028)$ of seminiferous tubules, were observed between short term experimental and short term control rats. However, a significant difference in right testis weight $(\mathrm{p}<0.001)$, left testis weight $(\mathrm{p}<0.001)$, volume of testis $(\mathrm{p}<0.001)$, diameter $(p<0.001)$ and area $(p<0.001)$ of seminiferous tubules was observed between midterm experimental and midterm control group. Similarly significant difference in right testis weight $(\mathrm{p}<0.001)$, left testis weight $(p<0.001)$, volume of testes $(p=0.038)$, and diameter $(p<0.001)$ and area $(p<0.001)$ of seminiferous tubules was observed between long term experimental and long term control group.

As compared to short term experimental group, midterm experimental group and long term experimental group had significantly lower right testis weight $(\mathrm{p}<0.001$ for both), left testis weight $(\mathrm{p}<0.001$ for both), diameter of seminiferous tubule $(\mathrm{p}<0.001$ for both) and cross-sectional area of seminiferous tubule $(p<0.001$ both). However, no significant difference were observed among midterm and long term experimental group in terms of testis weight, volume, diameter and crosssectional area of seminiferous tubules.

Figure 1 (A, B, C and D) shows the cross-section of seminiferous tubules of control and various experimental groups. It was observed that the crosssections of the seminiferous tubules of all the control groups were moderately circular or oval in outline with normal seminiferous epithelium and numerous spermatozoans within their lumen. The interstitial cells were also normal and prominent. Rats that were given doxorubicin showed progressive degenerations in their seminiferous tubules lumen. Group 1 rats showed a little reduction in the basal epithelial layer resulting in the apparent detachment of the basement membrane. The rats of group 2 (midterm experimental group) showed vacuolization of the cells, reduction in the number of spermatozoa. Lumen of the seminiferous tubules was significantly vacant. The interstitial spaces were distinct. Group 3 rats (long term experimental group) showed marked variation in its architecture. There was much testicular atrophy and most of the spaces were occupied by the interstitial cells.

Table 1. Changes in anatomical and morphometric parameters of albino rats

\begin{tabular}{|c|c|c|c|c|c|c|}
\hline Parameters & Group l & Control l & Group 2 & Control 2 & Group 3 & Control 3 \\
\hline $\begin{array}{l}\text { Initial body weight } \\
\text { (gm) }\end{array}$ & $163 \pm 10.59$ & $169.50 \pm 13.00$ & $168 \pm 10.85$ & $166.50 \pm 13.75$ & $161 \pm 10.48$ & $167 \pm 13.98$ \\
\hline $\begin{array}{l}\text { Final body weight } \\
\text { (gm) }\end{array}$ & $149.50 \pm 13.83$ & $177 \pm 10.32$ & $180=10.80$ & $188=19.74$ & $143.50 \pm 30.91$ & $181 \pm 13.70$ \\
\hline Weight difference & 13.5 & 7.5 & 12 & 21.5 & 17.5 & 14 \\
\hline \multicolumn{7}{|l|}{ Testis weight (gm) } \\
\hline Right & $0.99 \pm 0.24$ & $1.11 \pm 0.12$ & $0.53 \pm 0.14$ & $1.17 \pm 0.13$ & $0.43 \pm 0.11$ & $0.97 \pm 0.11$ \\
\hline Left & $0.94 \pm 0.28$ & $1.08 \pm 0.13$ & $0.48 \pm 0.16$ & $1.19 \pm 0.16$ & $0.39 \pm 0.12$ & $0.97 \pm 0.14$ \\
\hline Testis volume (cc) & $2.30 \pm 0.48$ & $2.50 \pm 0.52$ & $2.20 \pm 0.42$ & $3.50 \pm 0.52$ & $2.50 \pm 0.52$ & $3.00 \pm 0.47$ \\
\hline $\begin{array}{l}\text { Diameters of S. } \\
\text { tubule }(\mu \mathrm{m})\end{array}$ & $318.20 \pm 37.97$ & $352 \pm 24.33$ & $241.95 \pm 6.83$ & $348.05 \pm 23.33$ & $208.30 \pm 12.30$ & $347.30 \pm 40.33$ \\
\hline $\begin{array}{l}\text { Cross-sectional } \\
\text { Area of S.tubules } \\
\left(\mu \mathrm{m}^{2}\right)\end{array}$ & $80501.15 \pm 18421.97$ & $97683.04 \pm 13275.52$ & $45986.76 \pm 2623.45$ & $95478.66 \pm 12867.21$ & $34167.20 \pm 3993.63$ & $95833.65 \pm 21456$ \\
\hline
\end{tabular}




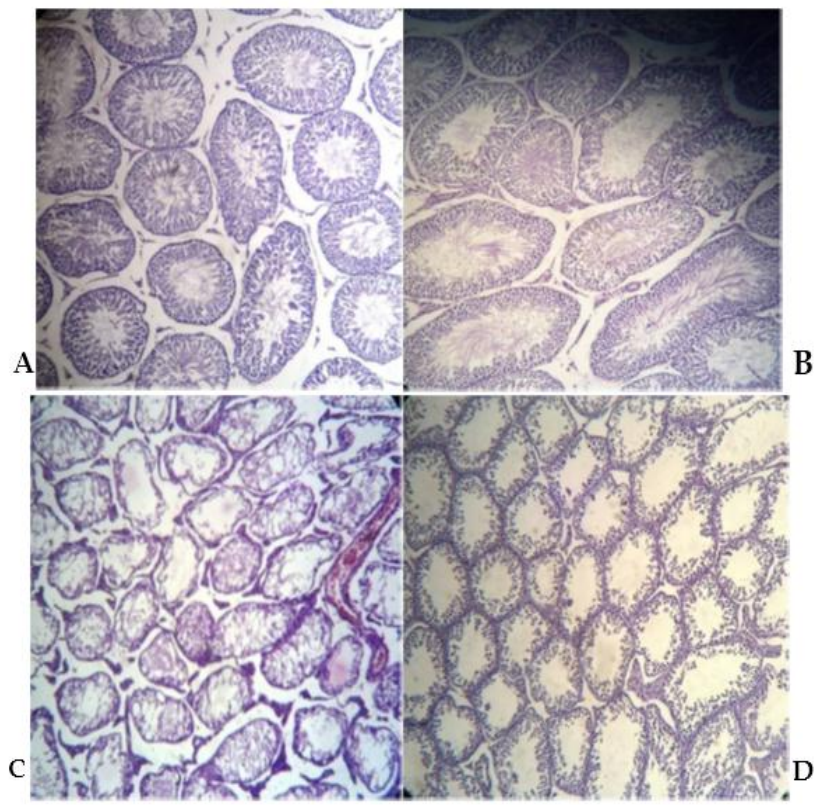

Figure 1. A, B, C and D show the cross-section of seminiferous tubules of control (all control groups), short term experimental group, midterm experimental group and long term experimental group respectively.

\section{Discussion}

Doxorubicin is one of the most widely used anticancer agents in the clinic despite its doselimiting side-effects. In the present study, we assessed the effects of a single dose (i.e. $10 \mathrm{mg} / \mathrm{kg}$ body weight of animal) of the drug on male albino rats. Present study shows that weight of the rats that were given doxorubicin decreases in comparison to the control groups. The decrease in body weight may be due to loss of appetite in experimental rats. We however observed that, the midterm experimental rats showed increase in body weight. The increase in body weight in midterm might be due to the reversible metabolic changes caused by the drug. After some period of time the body regains its metabolic function and then it again decreases due to the long term effect of the drugs.

In the present study significant difference in testicular weight among the midterm experimental and the midterm control groups, and among the long term experimental and the long term control groups was observed, which is similar to the findings of Saalu et al [7]. We also observed progressive decrease in testis weight from short term to long term experimental group. Previous study done by Howell et al. also reported that administration of doxorubicin can decrease the testicular weight of the rats. This could be due to the severe parenchymal atrophy in the seminiferous tubules following the doxorubicin administration [8]. However study by Prahalathan et al. did not report any significant testicular weight changes with doxorubicin treatment [9].

In the present study, we found that the diameter and cross-sectional area of seminiferous tubules decreases with the time in the rats given doxorubicin. As compared to short term experimental group, midterm experimental group and long term experimental group have decreased diameter and cross-sectional area of the seminiferous tubules, which may be due to more effect of the drugs on exposure to long time. Our findings are in accord to previous study by Saalu et al [7]. Study by Saalu et al. reported that doxorubicin chemotherapy induces morphological and morphometric impairments of testes of rats and progressive worsening of testicular derangement with time following a single dose of doxorubicin treatment [7]. Recent study done in India showed that treatment with doxorubicin alone caused decrease in body weight, sperm count and serum testosterone and increase in serum level of lactate dehydrogenase (LDH), creatine phosphokinase (CK), and glutamic oxaloacetate transaminase (GOT) [10].

In the present study, we observed deterioration in seminiferous tubules architecture in experimental group, and the architecture was more impaired in long term experimental group than midterm and short term experimental rats. The study also demonstrates that testicular cytotoxicity caused by doxorubicin provokes germ cell depletion in the seminiferous epithelium of the rats. Saalu et al. showed that even a single dose of doxorubicin progressively causes testicular derangement which may be due to its capacity to generate intracellular free radicals and reactive oxygen species [7]. Single higher doses or multiple lower doses of doxorubicin have been referred to be toxic to the stem spermatogonia, diminishing their survival [11]. The alteration of the measurements of testicular volume and weight, tubular diameter, seminiferous epithelium height and volume densities of tubular lumen and seminiferous epithelium can give information about the testicular damage degree as a consequence of germ cell death [12]. Franca et al. 
reported that when a massive germ cell loss occurs, it is followed by a sharp decline in testicular morphometric parameters [13]. In general, germ cell death caused by anticancer drugs, including doxorubicin, culminates with a reduction of morphometric parameters [14]. Many of doxorubicin dose-limiting toxicities occur due to its generation of toxic oxygen species, resulting in oxidative stress.

In summary, present study reveals deteriorative effect of doxorubicin on seminiferous tubules of rats. The study has however, several limitations. First, we did not assess the ultrastructural changes in testis caused by doxorubicin. Also, sperm count was not done in this study. The body weight of the experimental rats fluctuated with the duration. The exact causes for the fluctuation of body weight were not identified in this study.

\section{Competing interests}

None

\section{Author's contribution}

SKS, SK, DC, CBJ and SB designed the study. SKS, SK and DC conducted the experiments. SKS and SK wrote the manuscript. DC, CBJ and SB reviewed manuscript. All authors read and approved the final version of manuscript.

\section{Acknowledgements}

We kindly acknowledge B P Koirala Institute of Health Sciences for the support in the study.

\section{References}

1. Atessahin A, Karahan I, Turk G, Gur S, Yilmaz S, Ceribasi AO: Protective role of lycopene on cisplatin induced changes in sperm characteristics, testicular damage and oxidative stress in rats. Reprod Taxicol. 2006 21(1):42-7.

2. Rubin P, Louis SC, Lawrence BM, Paul O. Late effect of cancer treatment on normal tissues. Springer Verlog, Berlin, Heidelberg. 2008 1:109-30.

3. Yagmurcaa M, Erdoganb H, Irazc M, Songurd A, Ucare M, Fadillioglu E: Caffeic acid phenethyl ester as a protective agent against doxorubicin nephrotoxicity in rats. Clin Chim Acta. 2004 348(1-2):27-34.

4. Sawada T, Tamada H, Mori J: Secretion of testosterone and epidermal growth factor in mice with oligozospermia caused by doxorubicin hydrochloride. Andrologia.1994 26(3):151-3.
5. Quiles JL, Huertas JR, Battino M, Mataix J, RamírezTortosa MC: Antioxidants nutrients and adriamycin toxicity. Toxicology. 2002 180(1):79-95.

6. Kobayashi H, Urashima M, Hoshi Y, Uchiyama H, Fujisawa K, Akatsuka J: Testicular morphological changes in children with acute lymphoblastic leukemia following chemotherapy. Pediatrics International. 1996 38:640-3.

7. Saalu LC, Enye LA, Osinubi AA: An assessment of the histomorphometric evidences of doxorubicin-induced testicular cytotoxicity in Wistar rats. Int J Med Med Sci. 2009 1(9):370-4.

8. Howell ST, Shaler SM: Testicular function following chemotherapy. Hum Reprod. 2001 7:363-9.

9. Prahalathan C, Selvakumar E, Varalakshmi P: Lipoic acid ameliorates adriamycin- induced testicular mitochondriopathy. Reprod Toxicol. 2005 20:111-6.

10. Patil L, R. Balaraman R: Effect of Melatonin on Doxorubicin induced testicular damage in rats. 2009 1(3):879-84.

11. Jahnukainen K, Hou M, Parvinen M, Eksborg S, Söder $O$ : Stage-specific inhibition of deoxyribonucleic acid synthesis and induction of apoptosis by antracyclines in cultured rat spermatogenic cells. Biol Reprod. 2000 63(2):482-7.

12. Vendramini V, Sasso-Cerri E, Miraglia SM: Amifostine reduces the seminiferous epithelium damage in doxorubicin-treated prepubertal rats without improving the fertility status. Reprod Biol Endocrinol. 2010 8:3.

13. Franca LR, Russel LD: The testis of domestic animals. In: Martínez-Garcia F, Regadera J, eds. Male reproduction: a multidisciplinary overview. Churchill Communications, Madrid. $1998: 198-219$.

14. Stumpp T, Sasso-Cerri E, Freymuller E, Miraglia SM: Apoptosis and testicular alterations in albino rats treated with etoposide during the prepubertal phase. Anat Rec. 2004 279:611-22. 